REVIEW ARTICLE

\title{
New Management Systems and the Ambiguous Nature of Re- sources: A Qualitative Study about Healthcare Middle Managers
}

\author{
Véronique Haberey-Knuessi ${ }^{*}$ and Jean-Luc Heeb ${ }^{2}$ \\ ${ }^{1}$ Haute Ecole de Santé Arc, University of Applied Sciences of Western Switzerland, Switzerland \\ ${ }^{2}$ Haute école de travail social Fribourg, University of Applied Sciences of Western Switzerland, Switzerland
}

* Corresponding author: Véronique Haberey-Knuessi, Professor, Haute Ecole de Santé Arc, University of Applied Sciences of Western Switzerland, Espace de l'Europe 11, 2000 Neuchâtel, Switzerland, Tel: +41-32-930-1224, Fax: +41-32-9301213, E-mail: veronique.haberey-knuessi@arc.ch

The focus of this article is an empirical investigation using the Demerouti model to examine the stress factors affecting nursing managers, doctors and nurses in their work, and the resources available to them. A survey conducted by healthcare managers showed that despite being very highly motivated in their work, their engagement with it is sometimes incomplete. The study revealed that the professional reality of these managers has changed significantly in recent years, owing to the introduction of new public policies and the resulting major changes that have occurred in how healthcare institutions function. Thus, while the Demerouti model insists on the importance of achieving a balance between the demands of the job and the resources available, the actual daily lives of middle managers show that demands are very high while resources are sometimes insufficient or insufficiently valued. Here we consider the ambiguous nature of some of these resources which, although described as such, tend under the influence of various factors to become constraints exercising a detrimental effect on professional commitment.

\section{Stress Management}

Stress is a phenomenon that receives a great deal of attention from the media whether in the medical press, mainstream newspapers, scientific journals or the media as a whole. It has been recognised as causing numerous problems and there has therefore been much research aiming to improve our understanding of it, particularly with respect to occupational health. It was first defined in the 1950s by the psychologist Hans Selye and is characterised by a high psychological and physical burden, which can become a problem when an individual's resources are no longer able to compensate for the burden and re-establish balance. The resources that an individual uses to manage activates compensatory mechanisms to help it respond to these demands. If the stress lasts, however, the body's resources begin to be depleted and can no longer fully meet its needs a process of exhaustion begins and the stress becomes detrimental to the individual's health.

This understanding of stress leads to two potential types of action to stem the phenomenon. The first strategy involves attempting to do something about the actual cause of the stress, i.e. the context or the specific elements that result in the overload of demands that create the stress: workload, emotional weight, financial problems, and so on. The second strategy, on the other hand, focuses on the individual and his or her capacity to respond to stress in a positive manner, i.e. by mobilising the relevant resources. The research presented here uses Demerouti's Job Demand-Resources Model (JD-R Model) to understand the dynamics of what happens with these resources. We attempt to show how S. Hobfoll's thoughts on resources provide an valuable complement to Demerouti's model with respect to identifying all the various facets of the complex problem of stress. This will be particularly interesting in a management system that is itself a powerful generator of stress.

Citation: Haberey-Knuessi V, Heeb JL (2017) New Management Systems and the Ambiguous Nature of Resources: A Qualitative Study about Healthcare Middle Managers. Int Arch Nurs Health Care 3:071. doi.org/10.23937/2469-5823/1510071

Received: November 02, 2016: Accepted: May 29, 2017: Published: May 31, 2017

Copyright: (C) 2017 Haberey-Knuessi $V$ et al. This is an open-access article distributed under the terms of the Creative Commons Attribution License, which permits unrestricted use, distribution, and reproduction in any medium, provided the original author and source are credited. 


\section{Rationalisation, an Added Pressure}

When managers of health or social care organisations are faced with constraints - especially financial ones - they are compelled to establish rationalisation measures. Just like business operates in the industrial and commercial sectors, they have to optimise resources whilst reducing costs to a minimum. In order to control rapidly spiralling costs, healthcare institutions have been burdened with methods of regulation that involve stress-inducing budget restriction policies [1]. As a consequence, various measures and major organisational changes have been implemented which had a strong impact on the hospital and care context. These policies which all come under the new public management umbrella and emerged in the " 70 s are underpinned by management theories that originated in industry. They are aligned with principles like market orientation, decentralisation, cost reduction or managerialism to increase efficiency and effectiveness of public service organisations. In order to reach this goal 'lean management' is a frequently used strategy to rule organisations [2].

If it is to be able to compete, the organisation must think not only in terms of reducing costs by cutting jobs, but also in terms of improving performance and quality without increasing costs. Such strategies require good knowledge of existing resources so that they can be used to the best possible advantage. However, not only material issues such as better use of equipment or pooling of various support functions are relevant. They include also - and most significantly - human factors like matching the skills available to the objectives set, ensuring that the tasks required are suited to an employee's qualifications or employees engage with their work. In the '90s, a number of management trends were often connected with the 'lean management' philosophy originating from Japanese Toyotism. The aim of these trends was to develop more efficient systems of organisation. The concept of 'lean management' first appeared against a background of economic crisis. Its propensity for infiltrating every sector and every professional sphere, including the healthcare sector and its various institutions, has prompted us to try and improve our understanding of the phenomenon [3]. The word 'lean' is used to describe a management theory that aims to divest a process of anything superfluous to its needs, resulting in a system that produces just enough, just in time. To achieve this, every stage of the production process is closely examined with the aim of eliminating waste of any kind, particularly as regards time and procedures as this is seen as an obstacle to performance. According to Hohmann [4], 'lean' refers to a "system that aims to generate maximum added value as cheaply and quickly as possible by using just enough resources to give clients what they see as valuable". Managers have a specific role in this process, especially as regards implementing a long term strategic vision and orienting every action and decision in its direction. Managing this ongoing interface between short, medium and long-term objectives and the tasks carried out by employees on a day-to-day basis, this is the role of middle managers, in healthcare establishments. This role has changed significantly.

\section{Middle Managers, the Mainstay of New Public Management}

Before new public management was implemented, these nursing managers carried out primarily organisational tasks and were more or less hidden in the wake of the doctors. Nowadays, however, they have had to come out of the shadows and fulfil a key role in the establishment. While this new way of organising things has given managers new goals and offers interesting challenges, it is not without its problems.

In this style of management, managers are expected to be hands-on. They must be familiar with all the particularities of the terrain so that they can guide and support their teams effectively. With client satisfaction as its central focus in the context of hospitals, 'lean management' needs to have a long term strategy to ensure its actions last. It must also be able to anticipate new challenges to come. Governance facilitates the implementation of mechanisms that allow an organisation to address the objectives set within different time-frames. As the people responsible for implementing institutional strategies, middle managers have to try and reconcile the demands of the latter with the imperatives directly connected with the actual functioning of the various services. They try to achieve a consensus, whilst eliciting and encouraging change when it is inevitable. The aim of 'lean management' is to seek out any problems encountered during operational phases in real time so as to respond and undertake corrective measures as quickly as possible. To this end, middle managers are expected to find out about any problems or difficulties that may arise, while the employees are also encouraged to help find solutions and suggest improvements.

When seen from this theoretical angle, 'lean management' is an idealised version of how large establishments might be managed that is underpinned by a highly participative vision. However, it is clear from the work of various authors that this ideal is still a long way off, particularly in the many establishments which restricted the vision to the aspect of cost cutting [4-6]. When the focus is only on costs, everything is undertaken in order to root out non-essential expenditures, but the positive aspects of the policy - such as education and training, finding time for dialogue, joint knowledge building and experience-sharing - are left by the wayside. This is likely to result in a system which becomes ineffective [7] or even detrimental to health, because it generates significant stress in a variety of areas. Negative effects that have been demonstrated so far relate to physical issues, including accelerated work rates and increasingly repet- 
itive tasks impacting on blood pressure or Musculoskeletal Disorders (MSDs) [7].

But there is also a psychological impact: According to Bourgeois and Gonon [8], this new way of organising work is associated with loss of meaning. Especially standardisation of activity and acceleration of work rates have been recognized as sources of stress that result in absenteeism and loss of qualified staff [9]. Another consequence of the intensification of activity is that workers' discretionary power is reduced [10]. Paradoxically, however, the 'lean management' encourages increased autonomy for workers. According to some authors, while there is less overt control from the 'boss', it has simply been replaced by alternative means of control consisting of procedures, administrative regulations and other similar constraints [11].

In this kind of system, middle managers play a key role, which is why such positions are becoming increasingly common. They are coaches or leader-mediators who also have a supervisory function as they must ensure that the objectives set by management are met [7]. Such managers find themselves at the sometimes uncomfortable interface between their overburdened, discontented teams and the management's practically unattainable orders and objectives. The ever-increasing multiplicity of demands made on them in terms of service, quality, organisation and flexibility goes hand-in-hand with an increased number of responsibilities, which in turn has its own consequences in relation to stress [12].

The policy of continuous improvement that underpins new management styles rests on indicators that show whether or not the processes used achieve the expected outcomes. However, it also questions whether further improvements can be made. The resulting proliferation of dashboards with their many and various indicators contributes to increased anxiety amongst staff. Such indicators are often inflexible and distanced from the realities of day-to-day work and their rigidity may seriously hamper the flow of any professional activity [13]. This means that managers must try and find a balance between responding to the indicators and interpreting their relevance to what the actual work involves in the real world.

Clearly, the demands made on workers have increased significantly, but they have also increased with respect to the new professional class of middle managers. Faced with the obvious difficulties of trying to alleviate a professional situation that is impacted by significant budgetary issues and policies that are primarily cost-cutting exercises, it becomes of paramount importance to consider the problem from another angle: resources.

\section{Importance of Resources as a Compensatory Mechanism}

Resources play a key role when considering any activity because they help us understand, perform and assess what is to be done. For a long time they were seen purely as a way of balancing the workload, but they have since then been recognised as a fundamental element in motivational processes. They are also thought to contribute to our coping strategies by giving them a creative dynamic [14] and they affect the development and protection of other values [15].

People have a two-pronged response to stress. On the one hand there are our involuntary defence mechanisms and on the other our coping strategies which we consciously bring into play to help us to deal with the stressful situation [16]. The coping process can involve a cognitive, emotional or behavioural activity and is a response that draws on both our own personal resources and those of our surrounding environment [17]. Coping strategies help us to imagine that things could be different or that we could have a different impact on what happens, thereby making reality easier to understand and manage [18]. Within this coping dimension, our personal resources help us to develop an appropriate response.

The difficulties inherent in the current hospital context have a significant influence on people's health, particularly when stressful phenomena result in the failure of neurohormonal compensatory mechanisms and individuals must therefore suffer the physical and psychological consequences of such stress. This is another reason why a more far-reaching study of available resources and how they might be further developed appears to be of particular relevance.

For this reason we decided to use the Job Demands-Resources (JD-R) model put forward by Demerouti, et al. [19] as the theoretical framework for the research outlined below. This model highlights the fundamental role played by values in an individual's relationship with stress particularly with reference to stress in the workplace. The heuristic use of this model together with its broad perspective and flexibility makes it an appropriate choice in both research and practice.

The aim of the JD-R model is to combine two fields of research: research into stress and research into motivation. According to this model, constraints impact on stress and resources impact on motivation. The JD- $R$ model was validated in 2001 and is intended to be integrative and multifactorial. It is in line with those of Karasek [20] and Siegrist [21]. It allows the researcher to go beyond simply recording pathogenic or facilitating factors and functioning modes in order to examine the dynamic role played by resources. According to this model, a worker's well-being is directly related to the characteristics of the work-place which are viewed as demands and resources.

On one side lie the Job Demands, i.e. constraints the worker encounters in his or her professional life. Be they physical, social or organisational, their common denominator is that they all require personal in- 
vestment on the part of the worker concerned. Under certain conditions, they can lead to total exhaustion of physical and mental resources and cause a variety of physical and psychological symptoms. On the other side Demerouti's model focuses on the internal and external resources available to the worker named Job Resources. This refers to the physical, psychological, social and organisational resources that reduce the negative impact of the demands imposed providing a kind of protection and helping the person concerned to achieve the objectives set $[19,22]$.

According to Demerouti, the simultaneous existence of high levels of demand combined with insufficient resources becomes a major source of stress. Various pieces of research also show that people can react very differently to stress depending on the personal, social and organisational resources available when subjected to similar workloads [23]. The compensatory nature of the resources helps to protect people from stress and its harmful effects. Unlike the models referred to earlier, Demerouti sees the concept of resources in a much broader sense and not just as referring to resources that are specific to the work context. Here the dynamic role played by resources of every kind is emphasised and they are seen as motivating factors or factors contributing to job satisfaction [24]. According to the JD-R model, resources have an intrinsically motivating role in that they encourage a sense of personal fulfilment and an extrinsic role because they make it easier to perform the task. Thus, they stimulate engagement. This latter aspect is said to be of even greater importance when demands are high, but only in so far as the necessary resources are available [15]. Moreover, they would appear to be auto-stimulatory as the existence of resources promotes the development of new resources in a kind of virtuous circle [15].

Hobfoll's 'resources spiral' theory [25] postulates that people try to store up resources accumulating more and more so as to build up stocks to draw on when demands are high. Resources are a central element in terms of guaranteeing the well-being to which one aspire. Thus a pleasant working environment is a source of increased self-esteem and self-confidence as it offers possibilities of self-fulfillment. It will in turn act as a brake on the negative effects of excessive demands. Conversely, stress would seem to appear not just when there are insufficient resources, but also when one is faced with the probable loss of resources in the near or more distant future [26].

According to Schaufeli \& Taris [27], resources also alter an individual's perception of the features of his or her professional situation and its related constraints, particularly in terms of the positive effect they can have on his or her sense of effectiveness. From this point of view, they appear to play the part of a third variable that explains the relationship between an individual's perception of the characteristics of his or her working environment and the level of satisfaction experienced there.

However, as Schaufeli, et al. [27] pointed out, Demerouti's model is more a description than an explanation. Although a causal relationship has been shown between resources and constraints with their relative effects in terms of satisfaction or stress, no comprehensive analysis of this relationship with respect to resources is offered. The observation is purely a dichotomous description of resources and constraints, whereas in practice things are not always so clear-cut. Thus Nahrgang, et al. [28] showed that a demand that stimulates, though it can have a positive effect on workers, will become a source of stress with negative effects if it is too challenging. This prompts consideration of whether specific resources, depending on the intensity of their presence and the stresscircumstances in which they operate, might possibly turn them into stress factors themselves. This approach, moreover, corresponds with a failing consideration we detected when exploring the JD-R model [15]. This study seeks to address this omission by examining in more detail certain resources that are frequently deployed in the context of management styles like 'lean management'. It aims to understand to what extent resources can go on being resources rather than turning into constraints.

\section{Method of Data Collection and Analysis}

The empirical data come from a two year research programme conducted in French-speaking Switzerland between 2011 and 2013 in five hospitals of varying sizes: two local hospitals with fewer than 300 beds, two regional hospitals with between 300 and 1000 beds and a university hospital with over 1000 beds. The data presented in this article were collected from managers working in these organisations in a variety of different roles: doctor-managers, nursing managers, cross-functional healthcare managers, technical managers and administrative managers. Participation was entirely voluntary. The high percentage of manager participation in our investigation bears witness to the level of interest in this issue. An overall response rate of $70.7 \%$ was achieved.

The 942 participants were first asked to complete a questionnaire, after which a number of focus groups were conducted by the authors in the various institutions (12 focus groups in all, attended by a total of 108 managers). In terms of ethical considerations, those who took part were given all the guarantees inherent to a research programme before they participated in a focus group such as conditions of participation, possibility of withdrawal, confidentiality agreement, etc. Two to three focus groups were held in each institution, composed of managers from 10 to 12 different people each time. There were slightly more female participants in the focus groups - 61 women as against 47 men. Nearly 
two thirds were nurse managers, one third were doctors and the remainder were cross-functional, technical or administrative managers. All were aged between 25 and 57-years-old. The large majority were able to report quite significant experience of working in their field of management ( 7 to 27 years).

The focus lasted an average of two hours and a free discussion, guided by a semi-directive framework, allowed free exchanges which gave very rich results.

The specific aim of this research was to understand the impact of new management styles on managers' professional practice and, more particularly, on their job satisfaction and their health taking a descriptive approach [29]. In this respect, the research was intended to comprehensively examine how the people involved perceived their reality.

Because the most significant results were obtained in the focus groups, as they offered the opportunity to examine the various subjects in depth through especially rich exchanges, we chose to present here only these focus groups results. Only a few percentages will be mentioned of the quantitative phase which are particularly illustrating in some subjects.

All the focus group meetings were recorded with the agreement of those taking part,and the recordings transcribed for the purpose of detailed analysis by theme. Each focus group meeting was first examined on its own in order to determine the main themes, after which the themes from the different groups were collated to provide an overall template that enabled final categorisation [30].

\section{Results}

Nearly $60 \%$ of the middle managers reported a good level of job satisfaction. However, significant differences in motivation and commitment were revealed in this study. Even when work motivation levels were very high on the commitment scale [31], commitment levels were significantly lower. This suggests the presence of disruptive elements, given that motivation precedes engagement and is in fact the basis for it. There would seem to be an obstacle hampering the smooth transition from motivation to practical engagement.

The investigation revealed three main brakes on manager engagement: increased workload, very heavy responsibilities assumed despite only limited autonomy and high demands in terms of flexibility. This resources will be further commented together with resources as a major asset.

\section{Increased workload}

The size of the workload makes managers feel as if they never quite finish anything, because they are always in fire-fighting mode and therefore never able to go into a matter in depth. Managers identified increased workload as resulting in physical and psychological disorders.
This is sometimes accompanied by a mental load, for example when managers have to ask their teams to comply with a management decision they do not agree with or which creates an overload for employees who are already stretched to the limit. In the words of one such nursing manager: "Every day we see the distress people already suffer at work, we know what the changes will involve but there's nothing we can do".

In addition to the increased workload, ethical issues also weigh on managers' minds. Whether it is about substandard care or a mismatch between the values of the hierarchy and those of the team, this can have a significant impact on how a manager feels - several managers (physicians and nursing managers) said that such aspects of their work prevented them from engaging fully with it. Mental load may be expressed by physical symptoms (headaches, digestive disorders, etc.) but it can also lead to the frequent use of sleeping pills (15\% of participants) or the use of analgesics whether very frequently $(8 \%)$ or occasionally $(38 \%)$. In-depth investigation we drove among participants during the focus groups shows that physical and mental symptoms have increased since reorganisation and new management styles have been introduced. Contradictory instructions are a major source of stress for these managers.

\section{Responsibility and autonomy - high expectations}

Managers in nursing but also in medecine recognised that this new mode of organisation has given them a new type of responsibility. They are aware of their central role and their superiors acknowledge that they are a vital link in the chain of command when it comes to ensuring that their teams implement the decisions that the institution has made. The longest serving amongst them states that they now have a higher status than they did before the reforms were introduced and enjoy having a mission to complete with a certain degree of new autonomy. Nevertheless, they are not all convinced that the extra autonomy brings added value as they feel that it is accompanied by greater responsibility yet no extra help. This extra responsibility is a particularly weighty load in that it must be assumed within a delicate context comprising numerous uncertainties over which they have very little control. As pointed out by a nursing manager: "Middle managers are not allowed to have a point of view about what happens in terms of policy decisions. They are told what to do middle managers are quite simply muzzled".

The system's poor organisation is another reason why this extra responsibility can be so burdensome. Many managers, especially physicians, deplored the increasing lack of organisation that had accompanied the introduction of the new public management reforms. Where previously only one person was responsible, there is now an imposing array of line managers. A physician manager mentioned: "So when you have to make a decision, no matter how secondary, it has to go right 
up through the hierarchy and then come all the way down again - it can take months for something as trivial as a minor purchase". Diluting responsibility in this way means that "no-one feels responsible" (physician), which then leads to institutionalised inertia. The new managerial policies and the grouping together of healthcare facilities have resulted in far more management jobs and the various departments have been given different directorates in a system that has been described as "tentacular" (physician). A radiologist-manager described the almost "schizophrenic" pressures caused by being part of the medical directorate on a personal level while the radiology department itself was attached to a different directorate with a different set of objectives. In another establishment, closure of the diabetes outpatient department had forced the specialist nurses to join a polyclinic and provide healthcare procedures for which they were neither qualified nor motivated.

All these new responsibilities must be exercised within a context of just-in-time healthcare where the very heavy workload forces managers to work extremely busy shifts and long hours without enough rest periods. Indeed a young doctor talk of how hard it was to spend 70 hours a week in an oncology department working with patients whose suffering he was scarcely able to hear because he himself had reached his limit. An older doctor described the dismay engendered by the relatively recent introduction of a new policy whereby new staff are taken on without consulting him. This has resulted in the mass recruitment of very young, inexperienced nurses and a worrying increase in the number of critical incidents - some fatal - occurring in his department. The presence of just a few experienced professionals would ensure that complications in a patient's condition were recognised and appropriate action taken. But financial considerations took precedence.

\section{From instruction to flexibility and adaptation}

Staff reported that they were expected to continually adapt their behaviour in order to meet the new requirements, but this only results in exhausting the people who have to deal with the constant changes. In view of their responsibilities, managers also have to be highly flexible - not just in what they do and say but also regarding communication as managers are expected to be available at all times. Continually on the alert with breaks regularly interrupted by telephone calls, managers are under constant pressure. They feel that they are expected to answer the phone even if it has never been stated officially. The same can be said for emails during holiday periods. Many managers prefer to deal with them as they arrive in order to avoid ploughing through the huge number that have accumulated after just one week's holiday.

The greatest pressure, however, is doubtless connected with meeting objectives. Managers have to deal with specific objectives that are increasingly hard to achieve yet they can also see the problems likely to occur if they are not met. For example, one doctor explained that he is expected to see a certain number of patients during his clinic. If he does not meet this objective, he will not lose his job but "it will lead to a whole lot of minor consequences from refusing a training request to moving my office to an isolated room at the other end of the hospital or not being consulted when decisions are being made". As resources are limited they are given, he told us, "to people who will make the best use of them". This doctor therefore has no choice but to meet the imposed performance objectives.

Managers appreciate being given responsibility as long as they can exercise it in accordance with their values. However, this condition appears to be increasingly uncertain and a nursing manager described how difficult it is "not to be able to look after the team and having to neglect the most vulnerable". They all recognise that the managerial reforms have given them more professional autonomy when it comes to managing their teams for example, but they deplore the growing lack of managerial autonomy at a time when they are being given more and more management tasks to complete.

To sum up, middle managers are undeniably motivated with respect to their activities and the associated challenges. But the responsibility allocated to them must be accompanied by the resources needed for its implementation, true discretionary powers and an autonomy that is not designed solely with regard to efficiency criteria. In addition, while flexibility can offer an opportunity to explore new horizons and new practices, it damages team spirit and undermines recognition of professional skills by suggesting that anyone can turn their hand to any activity when imposed to excess.

\section{Resources, a major asset}

The great majority of resources are allocated as specific support for third parties. Support from line managers is highly appreciated even if the fact that this upper hierarchy has listened does not mean that the situation will change and problems will be solved. Simply having the opportunity to talk to one's line manager can be soothing. On the contrary, feeling that one's needs and perceptions are not being taken seriously engenders a feeling of malaise and frustration. Many managers said that they were unhappy with the lack of attention and recognition they receive from their superiors. Everyone questioned stressed that help and support from colleagues was of paramount importance and that it was difficult to implement decisions from management bodies without this special upholding, particularly if the decisions were not very well received.

Even the more popular aspects of these new roles such as project management and increased flexibility also tend to distance nurse managers from their teams as they become increasingly absorbed in a variety of 
cross-functional projects. This leads to a feeling of isolation that is hard to manage. Especially nursing managers experience this loneliness. As they are no longer involved in day-to-day nursing activities, they can quickly lose touch with the world of healthcare even though it was the original motivation for choosing their career. They describe a dual loss - they no longer have any contact with patients and they no longer feel part of a team - knowing as they do that their presence is not always welcome.

\section{Discussion}

Results of this qualitative study are about the healthcare managers' view on changes in their organisational setting and possible consequences on resources. As such, the study stresses the perception of the managers and not an objective causal link between new public management and work conditions. Managers reported changes which they attributed to requests of the hospital management. They located this changes within a context of reformed management policies. Thus, results shoud be unterstand according to Thomas' theorem [32]: since managers consider changes due to new management policies as real, their consequences on their working conditions are real. However, considering that Switzerland's healthcare system is increasingly and consistently governed by new public management rules [33], the managers' perception mirrors organisational changes, especially when they mention changes regarding chiefly workload, responsibilities or flexibility. Additionaly, managers' consistent perception across the five different hospitals they worked in suggest that their subjectivity echoes a common objective process of change. Furthermore, when looking at consequences, findings among nursing managers revealed that the type of hospital was not relevant to exhaustion [34]. Also, findings are in line with previous empirical research emphasising the relationship between managerial changes in hospitals and profesionals' health [35-37].

\section{Limitations of some resources}

According to Demerouti's JD-R model, resources are a catalyst promoting motivation and engagement particularly in the context of a demanding job. However, Demerouti and Bakker also talks about certain aspects that in fact become obstacles to these resources such as role conflicts, work overload and task ambiguity [15]. While the tasks allocated can be motivating factors, this only happens if they are balanced out by the appropriate resources.

To some extent, both autonomy and responsibility are beneficial dimensions. They provide managers with a certain degree of freedom and enable them to find meaning in their work, to prove their capacity and to obtain social recognition. But when a facilitating environment that promotes autonomy and responsibility is missing, they become constraints. Indeed, autonomy then turns out to be nothing else than a form of controlled freedom (for exemple by objectives that have to be achieved) and responsibility becomes burdensome because it is no longer in accordance with the means of the person.

According to Hobfoll [25], stress and disengagement arise in three situations: lack of sufficient resources, threatened lack of resources in the future and the impossibility of obtaining more resources. The present research shows that managers encounter the first situation less frequently, but encounter the second and third alternatives very much more frequently. The interviews conducted confirmed that fear of deteriorating working conditions significantly hampers engagement because of the anticipated problems. Moreover, owing to their threatening nature, people tended to assign greater importance to losses than to the resources themselves. The impact of losses are "rooted in cognition" [26]. For managers, the loss of autonomy in a context increasingly bound by protocols where monitoring takes place implicitly through multiple procedures and assessments results in a significant fear that their professional practice will suffer. This leads to demotivation by anticipation. Furthermore, the impact of the loss of resources depends on the value accorded to them in relation to one's culture or environment.

\section{Importance of personal and professionnal values}

In the healthcare world, collective values are of paramount importance [26]. Indeed, teamwork and peer recognition were two of the elements most frequently mentioned as sources of satisfaction or even as being the reason for someone's choice of career in the present research. It is therefore easy to understand why loss of the relational dimension with respect to the team, a knock-on effect of the new organisation systems and new missions might constitute a major depletion of resources. In this study, all the managers agreed that they were initially supported by their teams when organisational problems arose. However, their inability to improve working conditions undermined their legitimacy in the long term and sometimes resulted in their exclusion from the team.

Just like this relational value, managers also emphasised the importance of values associated with quality of care. Here too, they were reacting before the event as most of them said that at the moment they were still able to provide quality care. But they can see an imminent danger in a management system that puts quantity and efficiency before relational and holistic care that is the basis of their understanding of healthcare. There is no doubt that this is one of the principal causes of distress, exhaustion and resignation. The most flagrant example was given to us by one of the institutions where we conducted our investigation, currently being taken over by a large private-sector group. The managers bitterly described how the group made no attempt to 
hide that its aim was to "make money, not take care of people". Also, members of the medical staff should agree to be "formatted" in the group philosophy, i.e. profitable patients only, high-end accommodation, etc. if they wished to go on working there. They were told that if they could not adhere to this philosophy then they should leave.

\section{Quality of care, conditions of engagement}

These various elements explain the difference that we observed between managers' motivation levels and their engagement levels. They are still highly motivated but engage far less with their work because of declining or disappearing resources. Engagement is being replaced by a protective attitude resembling a retreat [15].

We have outlined how frustrating and even damaging it is for managers to feel unable to care about patients or teams according to one's values. They experience this impossibility as a personal failure and often feel guilty. Because this is source of suffering, many of them explained to have made a deliberate choice to restrict their commitment when compared with the past. They prefer to remain aloof even if it is sometimes considered as disengagement.

When considering resources, it becomes clear that they are not always objective or easy to evaluate. In fact they can be of a fundamentally subjective and symbolic nature. We cannot therefore focus solely on the loss of material resources. We must also take into account less visible resources and consider their quality and distinctive features. Similarly, assessing the resources available to an employee at a precise moment in time whilst ignoring their evolutionary character is extremely limiting. Demerouti and Bakker [15] recognise that the results of an investigation can be very different depending on whether the focus is the short or the long term, as the impact of resources takes place over a period of time - a factor that also affects their quality. From this point of view, Hobfoll [26] takes into account the effects of anticipated changes and enables us to fill the gap in the JD-R model which only takes a snapshot of the resources available at the time.

\section{Resources, a multifaceted concept}

Demerouti's JD-R model uses the concept of resources in its broadest sense, but it would be of interest to look at this notion in greater depth so as to fully understand their quality and particular features. What may indeed act as a resource in some situations may in others turn out to be a constraint. Thus, for example, autonomy and responsibility can be seen as a challenge by some persons in some situations and become a source of motivation. In other circumstances they become a burden exerting pressure and even resulting in exhaustion. By understanding the nature of these resources, managers would be able to assess both their scope and their limits.
Thereby, they could possibly establish a better balance with the demands made by their work. Acknowledging the value of resources is an important way for managers to recognise the efforts of their teams. Indeed, it shoud be kept in mind that lack of recognition is one of the principal causes of disengagement. Our research shows that such a quest is even more vital in so far as the new management styles are much more interested in results and process efficiency than in considering people and their needs. In such circumstances, factors that would normally be seen as resources run the risk of quickly becoming constraints if the fact that they involve human beings is not taken into account.

New public management is regarded as an opportunity to concentrate efforts and avoid wasting energy. It promotes synergies and opens up new possibilities. Managers are happy to take part in addressing such a significant challenge and the associated workload is not seen as a problem as long as they are able to take it on. In this respect, Demerouti's model allows us to see how the inherent values align with the constraints imposed. But the problem is caused by the nature of the resources themselves and how they fit into the timeframe. And this is where it appears to be of particular interest to link Demerouti's model with that of Hobfoll who focuses on these very aspects. When factors like responsibility and flexibility are customarily considered as resources, they can in fact become constraints, particularly if they are underpinned by different values. There is therefore much work to be done at management level to see resources not just in generic terms but also in terms of values.

\section{Conclusion}

It is no doubt unrealistic to imagine that there is such a thing as the perfect management system enabling all the tensions within an organisation to be resolved. Nevertheless, it is possible to seek ways of working together to build a future that has meaning for the individuals involved and fits in with a company's strategy and development at the same time. Strategies are key and 'lean management' is a tool for implementing them [4]. Where there is a common interest, a company can develop its full potential. We cannot overemphasise the importance of the virtuous circle. Where satisfied employees are motivated to engage with their tasks and therefore accomplish high quality work, this has in turn positive consequences for both the institution and the employees concerned in terms of recognition which will itself become a new source of motivation. However, diverging interests and unanswered problems open the door to the kind of double constraints that put people into quasi-schizophrenic conditions which will eventually have a detrimental effect on their health [38]. "Respect for the community means taking into account the fact that a company exists within a social framework that it must take into consideration" [38]. In addition to respect for 
the community, the issue here is about the central place of the individual at the heart of every production process.

While strategic management is based on the concept of value creation, this cannot be the sole purpose of an organisation's management policy. Value must be created 'with', 'by' and 'for' people and must include the elements that are seen to constitute 'value in the eyes of the professionals'. To take their vision of healthcare into consideration, it would seem appropriate for middle managers to be involved in managing the institutions where they work. If hands-on professionals were to take part in managing hospital structures, various pitfalls would doubtless be avoided. A dialogue could take place with respect to the values promoted by the different parties and a consensus could be sought that would be able to take into account the specific nature of healthcare and how it exists within a timeframe. More appropriate indicators than those based on purely performance-based criteria, which are quantified and evaluated solely in the short term, could be sought. This would mean that healthcare professionals feel that their philosophy received more recognition and therefore the dynamic would guarantee renewed motivation that could in turn be transformed into genuine engagement [27].

Demerouti's JD-R model can help management to understand the role played by resources and how to stimulate them, thereby helping to give healthcare professionals their rightful place. But it also highlights that they have limits and must be considered with reference to a timeframe that is in line with people's actual needs. Management will truly be able to influence employee engagement only if it takes these needs into account [39].

\section{Acknowledgement}

The research was funded by the Swiss National Science Foundation (grant 13DPD6_134764) and approved by the ethics committee of Lausanne University.

\section{Conflict of Interest}

No.

\section{References}

1. OCDE (2005) Maîtriser la hausse des dépenses publiques de santé. Études économiques de l'OCDE, 77-96.

2. Peters BG (2010) Nouveau Management Public. New Public Management. In: L Boussaguet, S Jacquot, P Ravinet, Dictionnaire des politiques publiques, Paris.

3. Ughetto P (2012) Le lean: pensée et impensé d'une activité sans relâchement. Activités 9 .

4. Hohmann C (2012) Lean Management: outils, méthodes, retours d'expériences. Paris.

5. Belorgey N (2011) L'hôpital sous pression. Enquête sur le nouveau management public. Paris.

6. Lawal AK, Rotter T, Kinsman L, Sari N, Harrison L, et al. (2014) Lean management in healthcare: definition, concepts, methodology and effects reported (systematic review protocol). Syst Rev 3: 103.
7. S Bruère (2012) Travail d'organisation du lean manufacturing et santé: à la source des risques. Perspectives interdisciplinaires sur le travail et la santé 14: 1-24.

8. Bourgeois F, Gonon O (2010) Le lean et l'activité humaine. Quel positionnement del'ergonomie, convoquée par cette nouvelle doctrine de l'efficacité? Activités 7: 136-142.

9. Rouzaud P (2011) Salariés, le lean tisse sa toile et vous entoure. L Harmattan, Paris.

10. Bouquin S, Stewart P (2009) Temps durs et dur labeur: un retour critique sur les modèles productifs de l'ère néolibérale. Actes des Journées internationales de sociologie du travail, Nancy.

11. Gorgeu A, Mathieu R (2003) Polyvalence, polycompétence ouvrières et intensité du travail: l'exemple de l'industrie automobile. Actes du Congrès changements organisationnels, gestion des resources humaines et communauté de pratiques. Compiègne: UTC.

12. de Coninck $F$ (2005) Crise de la rationalité industrielle et transformations de la prescription. Une étude de cas. Sociologie du Travail 47: 77-87.

13. Daniellou $F$ (2009) L'ergonome et les débats sur la performance de l'entreprise. Actes des 16èmes Journées de Bordeaux sur la pratique de l'ergonomie, Bordeaux.

14. Hobfoll SE, Buchwald P (2004) Die Theorie der Ressourcenerhaltung und das multiaxiale Copingmodell - eine innovative Stresstheorie. Stress gemeinsam bewältigen, Göttingen, 11-26.

15. Demerouti E, Bakker AB (2011) The Job Demands-Resources Model: Challenges for future research. SA Journal of Industrial Psychology 37: 580-589.

16. Cohen S, Kamarck T, Mermelstein R (1983) A global measure of perceived stress. Journal of Health and Social Behavior 24: 385-396.

17. Lazarus RS, Folkman S (1984) Stress, appraisal and coping. Springer, New York.

18. Bruchon-Schweitzer M (2002) Psychologie du travail. Modèles, concepts et méthodes. Dunod, Paris.

19. Demerouti E, Bakker AB, Nachreiner F, Schaufeli WB (2001) The job-demands resources model of burnout. Journal of Applied Psychology 86: 499-512.

20. Karasek RA (1979) Job demands, job decision latitude, and mental strain: Implications for job redesign. Administrative Science Quarterly 24: 285-308.

21. Siegrist J (1996) Adverse Health Effects of high Effort/low Reward Conditions. J Occup Health Psychol 1: 27-41.

22. Bakker AB, Demerouti E, De Boer E, Schaufeli WB (2003) Job demands and job resources as predictors of absence duration and frequency. Journal of Vocational Behavior 62: 341-356.

23. Bakker AB, Hakanen JJ, Demerouti E, Xanthopoulou D (2007) Job resources boost work engagement, particularly when job demands are high. Journal of Educational Psychology 99: 274-284.

24. Dollard MF, Bakker AB (2010) Psychosocial safety climate at a precursor to conducive work environments, psychological health problems, and employee engagement. Journal of Occupational and Organizational Psychology 83: 579-599.

25. Hobfoll SE (2001) The influence of culture, community, and the nested-self in the stress process: Advancing conservation of resources theory. Applied Psychology 50: 337-421.

26. Hobfoll SE (2002) Social and psychological resources and adaptation. Review of General Psychology 6: 307-324. 
27. Schaufeli WB, Taris TW (2014) A Critical Review of the Job Demands-Resources Model: Implications for Improving Work and Health. In: GF Bauer, O Hämmig, Bridging Occupational, Organizational and Public Health: A Transdiciplinary Approach. Springer, New York.

28. Nahrgang JD, Morgeson FP, Hofmann DA (2011) Safety at work: A meta-analytic investigation of the link between job demands, job resources, burnout, engagement, and safety outcomes. J Appl Psychol 96: 71-94.

29. Dumez H (2013) Méthodologie de la recherche qualitative, Paris.

30. Crabtree BF, Miller W (1992) A template approach to text analysis: Developing an using codebooks. Doing qualitative research, Sage Publications, Newbury Park, USA.

31. Meyer JP, Herscovitch L (2001) Commitment in the workplace: Toward a general model. Human Resource Management Review 11: 299-326.

32. Thomas WI, Thomas DS (1928) The child in America. Behavior problems and programs. AA Knopf, New York.
33. OECD (Organisation for Economic Co-operation and Development) and WHO (World Health Organization) (2011) OECD Reviews of health systems. OECD Publishing, Switzerland.

34. Heeb JL, Haberey-Knuessi V (2014) Health professionals facing burnout: what do we know about nursing managers? Nursing Research and Practice 2014: 681814.

35. Greenglass ER, Burke RJ, Fiksenbaum L (2001) Workload and burnout in nurses. Journal of Community and Applied Social Psychology 11: 211-215.

36. Norrish BR, Rundall TG (2001) Hospital restructuring and the work of registered nurses. Milbank Q 79: 55-79.

37. Haberey-Knuessi V, Heeb JL (2015) Medical heads and head nurse between managerialism and professionalism: a necessary double-bind? In: Winston TN, Handbook on burnout and sleep deprivation: risk factors, management strategies and impact on performance and behavior. Nova, New York.

38. Ballé M, Beauvallet G (2013) Le management lean. London.

39. Tims M, Bakker AB, Xanthopoulou D (2011) Do transformational leaders enhance their followers' daily work engagement? The Leadership Quarterly 22: 121-131. 